\title{
THE CALIBRATION OF THE "FINGERHUT" IONIZATION CHAMBER
}

\author{
By Lauriston S. Taylor and G. Singer
}

ABSTRACT

The use of the "thimble" ionization chamber (Fingerhutkammer) has in the past been the only method for comparing the primary X-ray standards of different laboratories, hence the precise calibration of two thimble chambers is discussed in this paper. Five principal factors determine the accuracy of such a calibration, and it is shown that the errors are all, in general, in the same direction. It is shown that the X-ray beam must have a uniform section sufficient to cover the thimble chamber and yet not be so diaphragmed that off-focus radiation may impair the readings of the standard chamber. The chamber must be carefully aligned in the beam experimentally and not merely centered on the basis of its geometrical shape, since the distribution of measured ionization is found to vary considerably over the length of the chamber. In the investigation of the standard ionization chamber ${ }^{1}$ the special conditions affecting the applicability of the inverse square law were described. The same general conditions were found to hold true for the thimble chamber, indicating the necessity of making a careful analysis of the diaphragm system before proceeding with the calibration. The "wall effect" of two thimble chambers was studied, and the very strong dependence upon quality of radiation indicated that any intercomparison by means of such a chamber will be valid only under identical conditions of voltage, filtration, and wave form of generator.

\section{CONTENTS}

I. Introduction

II. Energy distribution across sections of the standard $\mathrm{X}$-ray beam

III. The inverse square law for thimble chambers.

IV. Distribution of ionization in thimble chambers.-.

V. Saturation conditions in thimble chambers

VI. "Wall effect" in thimble chambers

VII. Summary

\section{INTRODUCTION}

A problem of fundamental importance in the standardization of $\mathrm{X}$-ray dosage is the comparison of the $(r)$ units established independently in the several national and local laboratories. It is ordinarily not possible or, perhaps, desirable to have every standardization equipment identical, and consequently any medium for intercalibrating the standards must be capable of being standardized accurately and unambiguously against each standard. Up to the present ${ }^{2}$ the only international comparison of X-ray standards has been through the use of the "Fingerhutkammer," 3 when Behnken carried such a calibrated chamber to several laboratories, measuring the $(r)$ unit as determined by each. The discrepancies in the cali-

1 L. S. Taylor, B. S. Jour. Research, 2, p. 771; 1929.

2 H. Behnken, Strahlentherapie, 29, p. 192; 1928.

3 The more common designation of the small chambers used in connection with dosage meters is the original German term "Fingerhutkammer." In the future we shall use the term "thimble" chamber for this device. 
brations against the respective standards were then used as correction factors when necessary.

The next problem of practical value is the calibration of the small portable ionization chamber or dosage meter. Since most biological reactions are seldom reproducible to within a small percentage, an accuracy of about 4 per cent is usually sufficient for the calibration of a dosage meter which is to be used for medical purposes. If a similar instrument is to be used for comparing standards, the accuracy of calibration should then be 0.5 per cent or better.

In some preliminary investigations it was found, however, that to attain an accuracy of 1.0 per cent in calibrating a small ionization chamber considerable care was necessary and, moreover, that differences up to several per cent might easily arise due to differences in technique used by different investigators. Consequently a study was made of certain details in the methods for calibrating the thimble ionization chamber and of the probable sources of error involved. For most of the errors it is not deemed necessary to make corrections inasmuch as special technique permits them to be avoided. We will point out the sources and magnitude in the case of several of the errors without attempting to provide corrections except in method.

In two earlier papers ${ }^{45}$ several possible sources of error in the standard ionization chamber were indicated, and it was suggested that they were of such a nature as to admit of the possibility of counterbalancing each other. The magnitude of some of them was determined and the experimental arrangements of several other observers were analyzed for the presence of such errors, which were, in general, found to be small. In the present work it has been found that the errors are not so likely to counterbalance each other but tend rather in the same direction; that is, they are such that the thimble chamber measures too little ionization.

In this study the standard ionization chamber of the Bureau of Standards was used as a reference for all measurements. A number of different types of dosage meter were available, ${ }^{6}$ of which two were chosen as being representative of all those employing the more commonly used thimble chamber. Consequently our study was confined to the Friedrich and the Glasser chambers, each, however, being used in conjunction with the same string electrometer.

\section{ENERGY DISTRIBUTION ACROSS SECTIONS OF THE STANDARD X-RAY BEAM}

The most important requirement for the standard X-ray beam is that it be uniform over the entrant diaphragm of the standard ionization chamber. ${ }^{78910}$ Beam uniformity over the area of the thimble chamber is likewise the outstanding requirement in the comparison against the standard. Below are given a number of curves showing the energy distribution over a section of the X-ray beam, illustrating correct and incorrect conditions.

\footnotetext{
4 L. S. Taylor, B. S. Jour. Research, 2 (RP 56), p. 771; 1929.

5 L. S. Taylor, B. S. Jour. Research, 3 (RP 119), p. 803; 1929.

6 Other dosage meters available were of the following types: Furstenau, Müller, Küstner "Eichstandgerat," Solomon, and Wulf. Of these the Furstenau and Küstner instruments do not involve the use of a "Fingerhutkammer"

7 J. S. Taylor. (See footnote 5.)

8 W. V. Mayenord, Brit. J. Rad., N. S. I., p. 125; 1928.

- Otto Glasser and U. V. Portmann, Am. J. Roent., 19, p. 47; 1928.

10 H. Behnken, Strahlenthørapie, 26, p. 79; 1927.
} 
The presence of the limiting diaphragm near the tube complicates matters somewhat. It has been found, however, that for standard ionization-chamber measurements such a diaphragm near the tube has certain advantages in avoiding the effect of off focus ${ }^{11}$ and stem radiation, although its use necessarily makes the uniform area of the beam small. As a consequence, if we have a thimble chamber whose length is from 1.5 to $4 \mathrm{~cm}$., it is clearly possible that, while the X-ray beam may be sufficiently uniform over an area necessary for the standard, it does not necessarily satifsy the requirements of the small chamber except at comparatively great distances from the tube. On the other hand, if the beam uniformity is made to satisfy the small chamber by, say, enlarging the limiting diaphragm next to the tube, then an error may be introduced in the standard determination due to off-focus radiation. It is evident then that the two measurements must be carefully made with proper regard to these conditions if an accurate calibration is to result.

In calibrating a small chamber the various methods used may be reduced to four, as indicated diagrammatically in Figure 1. The first $^{12}$ (position 1) places the thimble chamber between the X-ray

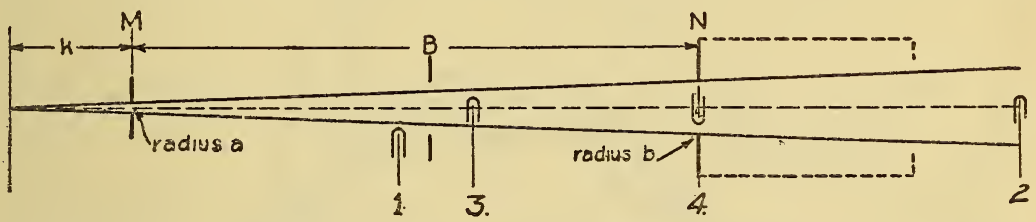

FIgURE 1.-Arrangement of diaphragms and thimble chambers for calibration

tube and standard ionization chamber $N$, and sufficiently off the axis of the X-ray beam so that it does not intercept the part of the beam entering the standard chamber. The ionization currents are then measured simultaneously and the inverse square law applied to the two. This is the system used by Behnken and, as seen, assumes that the beam is uniform over a comparatively wide area. In the second method (position 2) the thimble chamber is beyond the standard and on the same axis; the readings are made simultaneously and the inverse square law applied. The third (position 3 ) involves a substitution method, the thimble chamber being inserted between the tube and the standard on the axis of the beam which is utilized by the standard; the readings are taken separately and the inverse square law applied. The fourth (position 4) also involves a substitution method, ${ }^{13}$ the thimble chamber being inserted in the position occupied by the front diaphragm of the standard and the use of the inverse square law thus avoided-a very desirable feature. The first two methods have the advantage of simultaneous measurement, thus minimizing the effects of an unsteady source of radiation. The choice of any of the methods will depend upon the beam uniformity which, in turn, depends on the limiting diaphragm $M$ and the applicability of the inverse square law. The last method is used by the Bureau of Standards since an extremely steady X-ray equipment

11 Off-focus radiation is defined as that radiation from an X-ray tube which originates from points on the target face other than the sharply defined focus.

12 H. Behnken. (See footnote 10.)

13 Otto Glasser and U. V. Portmann, (See footnote 9.) 
conurolled by a specially constructed voltage stabilizer is available. In this equipment the standard and small chamber are fixed in position while the X-ray tube and beam are shifted laterally from one to the other, keeping the relative position of target to chamber the same for both.

The uniform distribution of energy across the beam may be tested most easily by means of an ionization chamber. Photographic determinations are more accurate if carefully executed, inasmuch as there is no effective slit width for which allowance must be made. Figure 2 shows for several diaphragm combinations and distances from the $\mathrm{X}$-ray tube, the intensity of ionization measured by the standard chamber as the beam is moved laterally across the entrant diaphragm $N$. (The curves are plotted on different scales so that the relative ordinates are without significance.) For all of this study the focal

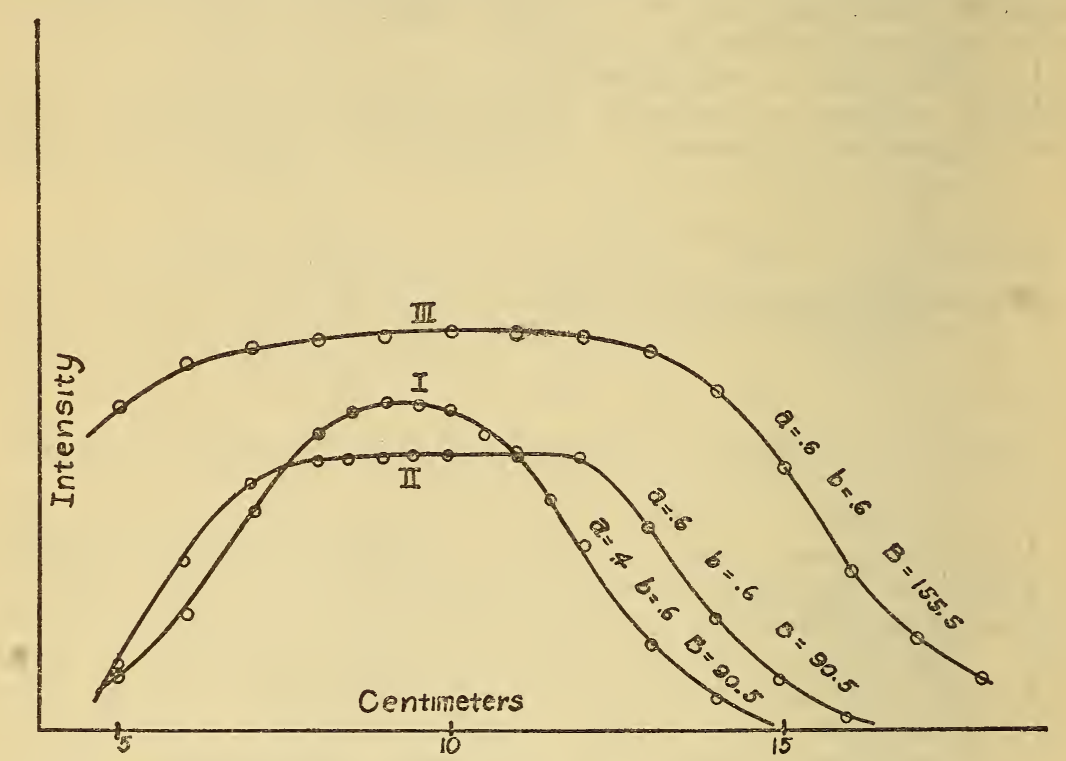

FIgURE 2.-Intensity distribution across standard X-ray beam. (Measured with standard chamber)

spot had a radius of about $0.6 \mathrm{~cm}$ and the diaphragm $M$ likewise a radius of $0.6 \mathrm{~cm}$ except where otherwise indicated. In Curves I and II the distance from tube to chamber was $90.5 \mathrm{~cm}$ while the radii of the limiting diaphragm $M$ were 0.4 and $0.6 \mathrm{~cm}$, respectively. In Curves II and III, both diaphragms had $0.6 \mathrm{~cm}$ radii and the distances were 90.5 and $155.5 \mathrm{~cm}$, respectively. It will be noticed that under the conditions of Curve I the peak of the curve is relatively narrow, hence, proper alignment of the system is very necessary so that, if possible, such conditions are avoided. By increasing the size of diaphragm $M$ to a radius of $0.6 \mathrm{~cm}$ (Curve II) the peak is considerably broadened though it is not of uniform intensity over its width. However, the increase in $M$ may be undesirable in that it allows "off-focus" radiation to enter the standard chamber. Curve III is similar to II except for having a broader peak. It is also of interest to note that all 
curves are very nearly symmetrical. This indicates a correct adjustment of the limiting diaphragm $M$, since if $M$ were out of alignment, off-focus radiation would show up as a dissymmetry. After correcting for the diameter of the large diaphragm the measured width of the peaks agree fairly well with the calculated widths.

The calibration of a thimble ionization chamber placed in the X-ray beam under conditions corresponding to Curve I would be in doubt if not in serious error since the X-ray intensity would not be uniform over the length of the chamber. Placed in the beam corresponding to Curve II the flux density of the beam would be sufficiently uniform, but due to the very large diaphragm $M$ used in the standard system an error due to off-focus radiation is introduced in the standard which rules out such an arrangement. A calibration of the fourth type, however, would be satisfactory under conditions corresponding to III,

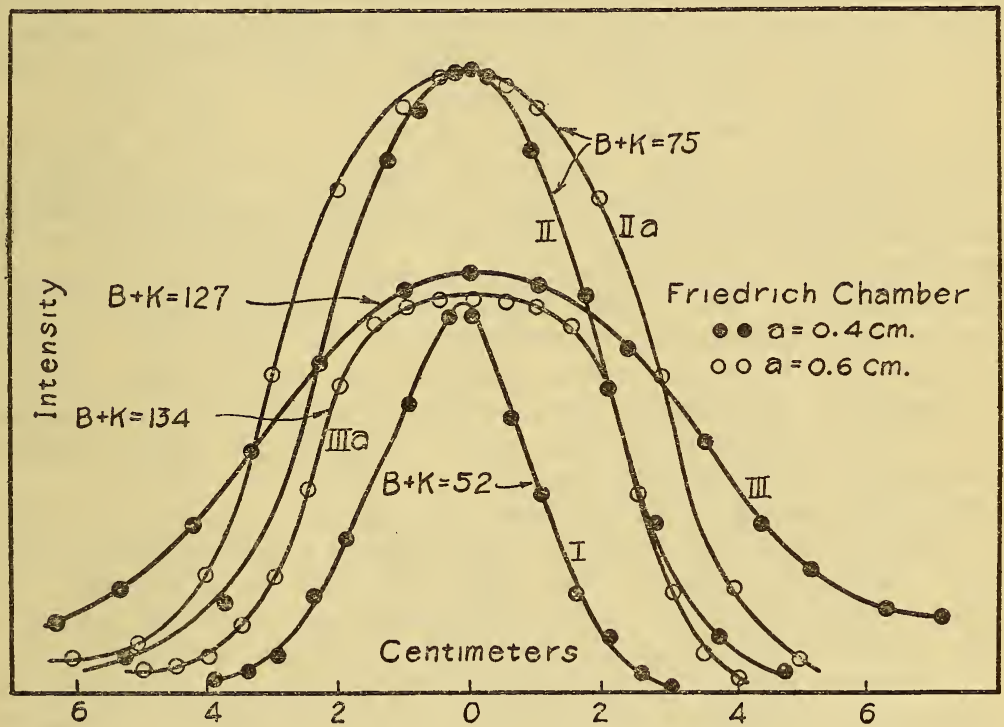

FIgURE 3.-Intensity distribution across standard X-ray beam. (Measured with thimble chamber)

in that the beam is uniform over the length of the thimble chamber and at the same time off-focus radiation is excluded from the standard chamber.

If now, using a thimble ionization chamber (Friedrich in this case), we measure the energy distribution across the beam the symmetry usually disappears and the width of the peak is apparently diminished. In general, any dissymmetry is due to the construction of the thimble chamber, which is such that the measured ionization is not uniform over its length. The apparent narrowness of the peak is caused by the large "effective slit width" of the Friedrich chamber, the actual uniform part of the beam being some $2 \mathrm{~cm}$ greater in diameter:

Figure 3 gives a set of such energy distribution curves under several different sets of conditions; Curves I, II, and III being taken with a limiting diaphragm $M$ of diameter $8 \mathrm{~mm}$ and curves IIa and IIIa with a limiting diaphragm $M$ of diameter $12 \mathrm{~mm}$. With the 
standard chamber at the same relative positions as indicated for the thimble chamber and the diaphragm $M$ having an $8 \mathrm{~mm}$ diameter (first case) the off-focus radiation is definitely excluded and an accurate standardization of the beam may be effected. With the diaphragm $M$ having a $12 \mathrm{~mm}$ diameter (second case) off-focus radiation enters the chamber and impairs the results.

\section{THE INVERSE SQUARE LAW FOR THIMBLE CHAMBERS}

In a paper referred to above ${ }^{14}$ it was shown that depending on whether the target, as viewed from the position of the chamber diaphragm, does or does not fill the aperture of the target diaphragm the inverse square for computing intensity is applied, respectively, to the target diaphragm distance, or the target distance. Thus, when the ionization chamber diaphragm filled the aperture of the system, ${ }^{15}$ the distance must be measured between the limiting diaphragm $M$ and the chamber diaphragm $N$. This condition becomes more important when using large ionization chamber diaphragms.

Since, to obtain sufficiently large effects, most thimble ionization chambers subtend a fairly large solid angle as measured from the focus, the inverse square law was investigated in a manner similar to that used for the standard chamber. The thimble chamber and electroscope tube were mounted so as to move along a track $2 \mathrm{~m}$ long placed parallel to the X-ray beam. The vertical and horizontal alignment was determined by measurement as above, the chamber being placed in the center of the uniform area of the beam. Ionization currents in the thimble chamber were measured for various settings of the chamber along the track, the position being measured with reference to the diaphragm $M$.

Plotting the intensity (I) against the square of the reciprocal distance $\left(1 / B^{2}\right)$ it is seen in Figure 4 that there is a distinct break in the curves. Three such curves are given; curve $\mathrm{G}$ for a Glasser $1 \mathrm{~cm}^{3}$ chamber, and Curve $\mathrm{F}$ for a Friedrich chamber; the first having an outside length of $2.9 \mathrm{~cm}$ and the second an outside length of $2.7 \mathrm{~cm}$. It will be also seen that the break in curve $\mathrm{G}$ occurs at a position corresponding to a distance farther from the tube than the break in curve F. Likewise the curves for both Friedrich and Glasser chambers break at a point farther from the tube than a similar curve $\mathrm{S}$ for the standard chamber. It is seen that the lower portion of each curve passes through the origin, indicating that the inverse square law is obeyed only beyond a certain distance from the X-ray tube. Also on the curve $G$ it is seen that the points 1,2 , and 3 are far off the straight portion covering smaller distances. This divergence is caused by approaching too closely to the tube where the beam intensity varies very rapidly across the diameter occupied by the chamber. (See Curve I, fig. 3.)

If we replot these same data as $\log I$ against $\log B$, we may determine the exact divergence from the inverse square law. (Fig. 5.) If the inverse square law is obeyed, the points should lie along a straight line having a slope of -2.0 . Actually it is seen that for a Friedrich chamber only the portion $b F$ has a slope of approximately -2.00 , for

14 L. S. Taylor. (See footnote 5. )

15 The aperture of the system is defined as the solid angle subtended by the focal spot and the limiting diaphragm $M$. (Fig. 1.) 


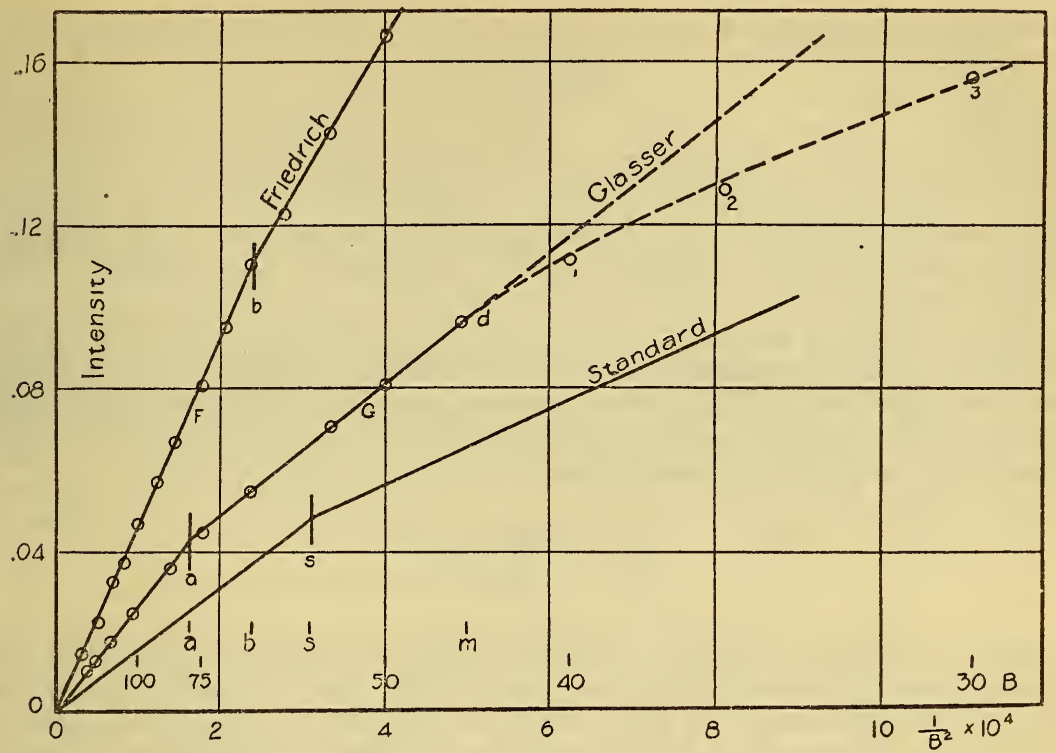

FIGURE 4.-Divergence from the inverse square law for three ionization chambers

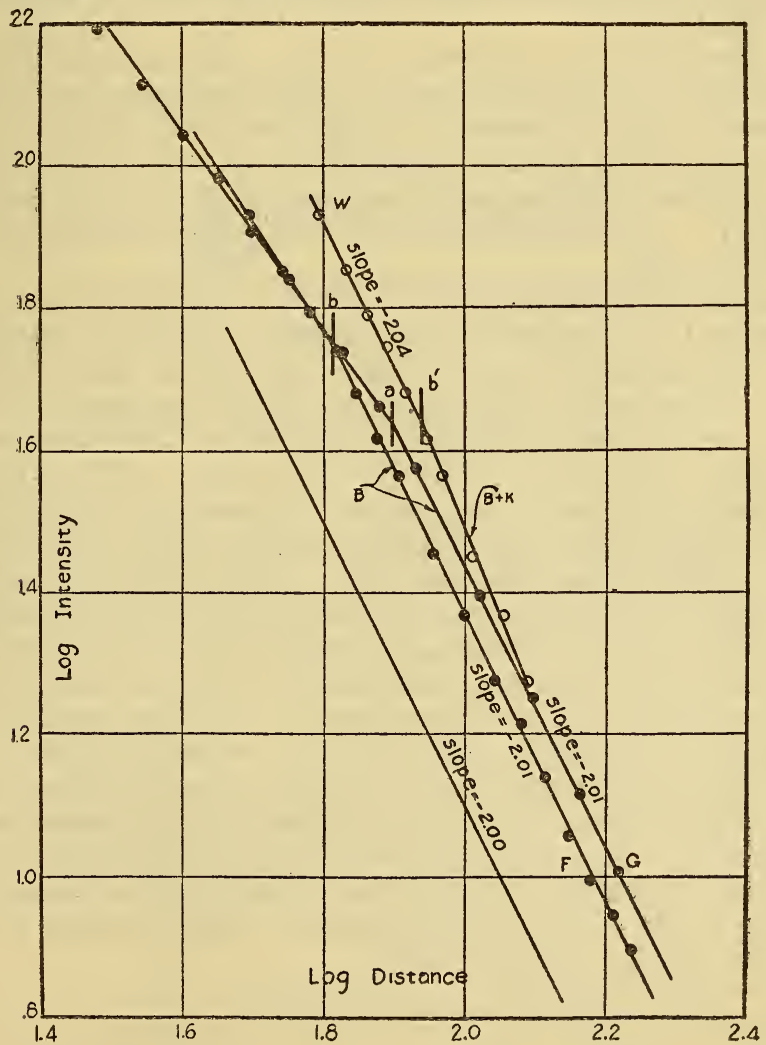

FigURE 5--Inverse power law for three ionization chambers 
a Glasser chamber the portion $a G$; these points corresponding to distances far from the tube. If we plot log $I$ against $\log (B+k)$ for the Friedrich chamber, it is seen that the part $b^{\prime} W$ most nearly approaches the slope -2 (being actually -2.04 ), corresponding to distances near to the tube. It is important to notice that the inverse square law is not obeyed exactly over any of the range of $(B+k)$. This bears out the conclusion drawn in an earlier paper ${ }^{16}$ that the use of an extended. source of radiation appears to be preferable for precise X-ray standardization.

By analogy to the case of the standard chamber, where now the thimble chamber replaces the standard chamber diaphragm, we should expect a divergence from the inverse square law at the position where the thimble chamber no longer fills the aperture of the system. ${ }^{17}$ This should be at such a distance that

$$
f=\frac{k a+k b+a B}{B}
$$

where $f, a$, and $b$ are the respective radii of focal spot, limiting diaphragm and standard chamber diaphragm (half the effective length of the small chamber in this study) and $B$ and $k$ are as indicated in Figure 1. However, it is impossible to determine the effective length of the chamber accurately, hence, merely the order of magnitude of $B$ can be calculated.

Having observed such a discontinuity in the intensity curve for the thimble chamber, we are confronted with the question of comparing the ionization measurements of standard and thimble chambers. Curve "Standard" in Figure 4 is for the standard chamber when using the same limiting diaphragm $M$ and an arbitrary standard chamber diaphragm $N$. It is seen that the break point for this curve differs from the first two.

Referring to Figure 5 we find when the thimble chamber is used at such distances that the inverse square law is applied to the diaphragm $M$, that the exponent of the distance is -2.01 , thus differing from -2.00 by 0.5 per cent. On the other hand, for such distances that the inverse square law is applied to the target the exponent is about -2.04 , giving a difference of 2.0 per cent from -2.00 . Correspondingly, we find when using a standard in place of a thimble chamber that the divergence of the respective exponents agrees in order of magnitude with the thimble chamber. Thus, if one chamber is calibrated against the other over regions where their exponents agree, the errors introduced will neutralize each other. However, if the calibration is made over regions where the two exponents differ, there will be an error of about 1.5 to 2.0 per cent. In other words, for calibration purposes, conditions should be so selected that either both chambers fill the aperture of the system or neither chamber fills it. Thus for the Glasser chamber the comparison should be made in the regions $O$ to $a$ or $s$ to $m$ and not in the region $a$ to $s$. When calibrating dosage meters for medical uses the errors introduced as above may not be serious, whereas for standardizing purposes their magnitudes warrant proper consideration. 


\section{DISTRIBUTION OF IONIZATION IN THIMBLE CHAMBERS}

In studying the general behavior of a thimble chamber it is important to know the distribution of ionization in the chamber itself. (See Sec. II.) For instance, it was found when measuring the intensity distribution across the beam that the peak of the intensity curve did not always coincide with the position of the geometrical center of the particular chamber used to determine it. To check this the beam center was obtained accurately by means of a Glasser $1 / 20 \mathrm{~cm}^{3}$ chamber and the geometrical center of the larger $(1 \mathrm{ml})$ chamber set at this point, after which it was usually found that the $1 \mathrm{ml}$ chamber had to be shifted slightly in order to obtain the maximum ionization reading. This means that we can not rely upon the geometrical center as the true center point in aligning the chamber for calibration.

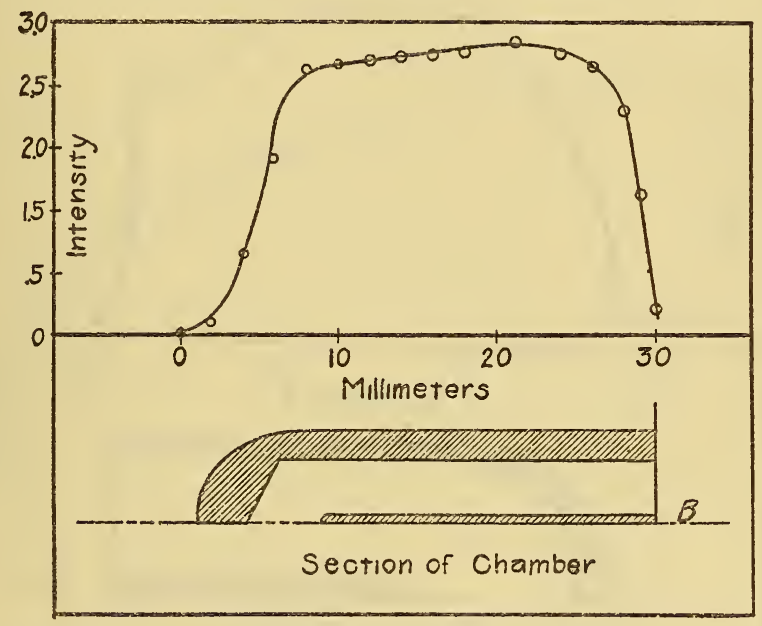

FIGURE 6.-Distribution of ionization along axis of Glasser chamber

To measure the distribution of ionization in the thimble chamber (and likewise to obtain the effective center), the X-ray beam was defined by a long slit about $1 \mathrm{~mm}$ wide and the chamber moved across this beam by small steps in a direction parallel to the chamber axis and at right angles to the long axis of the slit, so that at any position only a small portion of the chamber air volume was ionized by the direct beam. Figures 6 and 7 show the distribution of ionization in a $1 \mathrm{~cm}^{3}$ Glasser and a Friedrich chamber, respectively. Below each curve is a half-section scale drawing of the corresponding chamber. In the case of the Friedrich chamber the center of the ionization peak agrees fairly well with the geometrical center. However, due to the very uneven ionization at the ends it is difficult to predict the true effective center when used in a broad beam. There is a marked increase in the ionization at the end of $B$ of the chamber, this being due to the metal collar which supports the chamber cap. The case of the $1 \mathrm{~cm}^{3}$ Glasser chamber is similar, although the center of the ionization peak is farther from the geometrical center while the scattering at the end $B$ appears to be negligible. 
As pointed out in Section II, it is not ordinarily possible to calculate the break point in the inverse square law from the measured length of the thimble chamber; however, having found the break point we may attempt to calculate the effective length of the chamber by reversing the process. A comparison of the values given in column 3 of Table 1, obtained in this way, with the over-all geometrical length given in column 2 shows at once the futility of trying to calculate the working range for standardization on the basis of the measured chamber length. The fact that the experimental length given in column 4, obtained by measuring the width of the peaks in Figures 6 and 7, is the same for both the Friedrich and Glasser chambers would

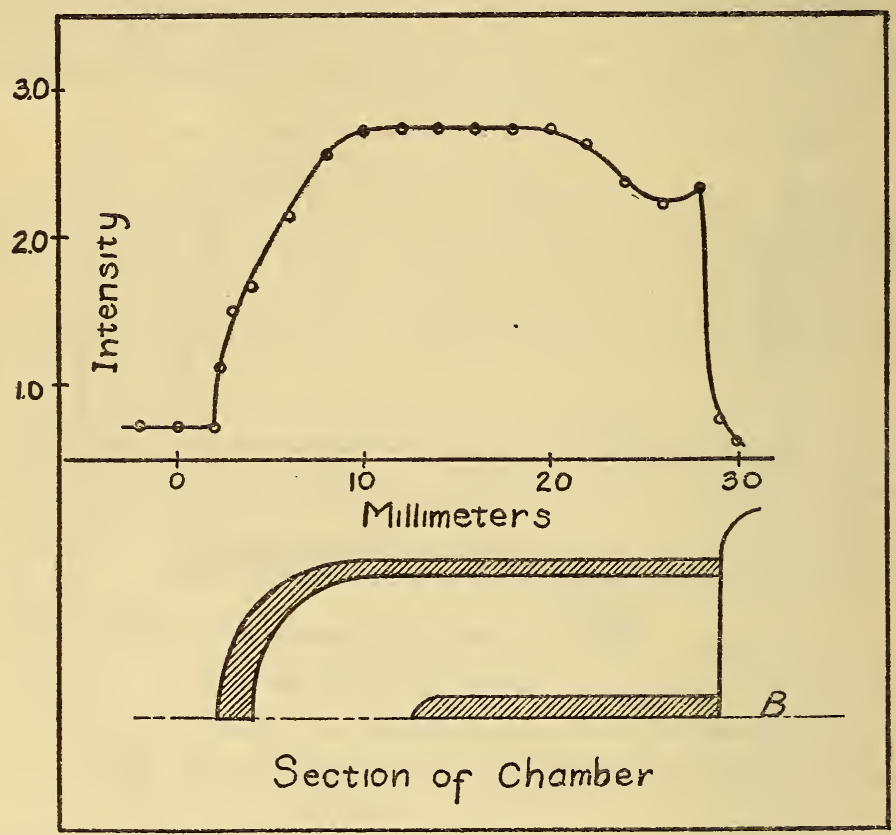

FIGURE 7.-Distribution of ionization along axis of Friedrich chamber

lead to the conclusion that the working range of the inverse square law was the same for both, and yet Figure 4 shows that their break points differ by $8 \mathrm{~cm}$.

TABLE 1

\begin{tabular}{|c|c|c|c|}
\hline Chamber & $\begin{array}{c}\text { Measured } \\
\text { length }\end{array}$ & $\begin{array}{c}\text { Calculated } \\
\text { length }\end{array}$ & $\begin{array}{l}\text { Experi- } \\
\text { mental } \\
\text { length }\end{array}$ \\
\hline 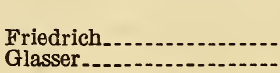 & $\begin{array}{l}c m \\
2.7 \\
2.9\end{array}$ & $\begin{array}{l}c m \\
2.5 \\
2.1\end{array}$ & $\begin{array}{l}c m \\
2.0 \\
2.0\end{array}$ \\
\hline
\end{tabular}




\section{SATURATION CONDITIONS IN THIMBLE CHAMBERS}

When a small ionization chamber is used with a medical-dosage meter the system is always charged to a potential of the order of 300 to 500 volts, thus assuring complete saturation of the chamber. In such cases the working sensitivity is comparatively low, requiring very long exposures for the radiation ordinarily used in treatmentan undesirable feature when using the small chamber for an intercomparison between two standards, since the likelihood of severe fluctuations in beam intensity is greater for long time intervals. As a consequence lower charging voltages (50 to 100) are frequently used, thus increasing the working sensitivity of the system. ${ }^{18}$ It is questionable whether there is always complete saturation under such conditions. ${ }^{19} 20$

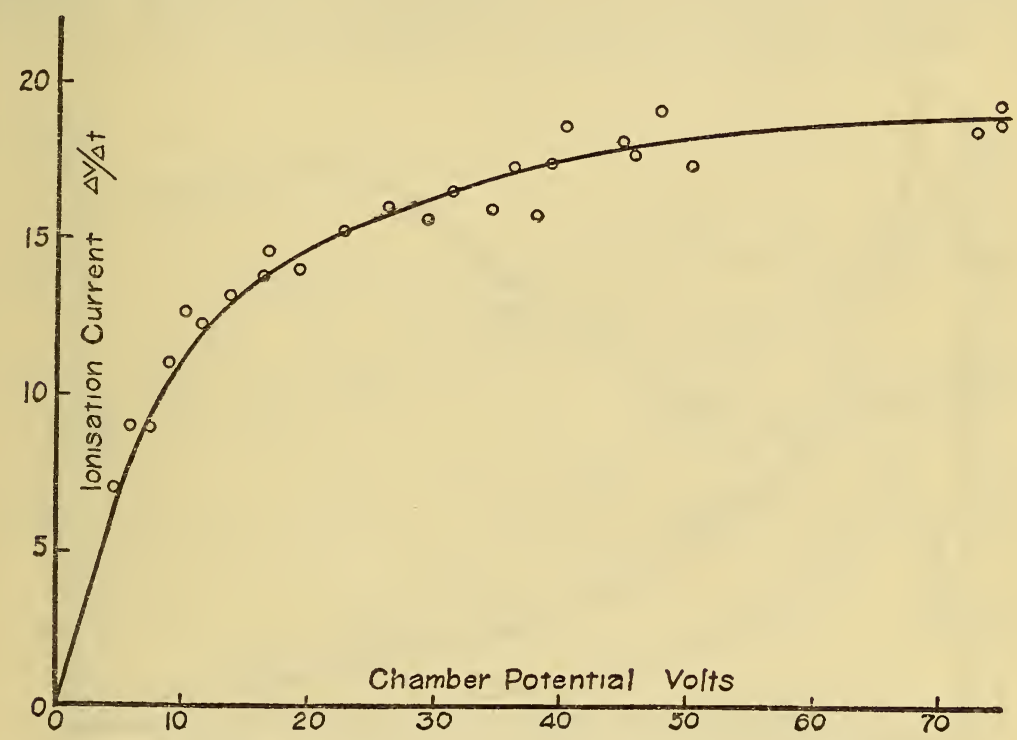

Figure 8.-Voltage saturation curve for thimble chamber

To test this a system was arranged so that a full-scale deflection of the electrometer corresponded to a potential of about 50 to 100 volts on the ionization chamber electrode. With the chamber exposed to a steady source of radiation, times of transit were measured for, say, every five divisions on the electrometer scale. Plotting time against the voltage corresponding to each scale reading a straight line would indicate uniformity of measured ionization; that is, saturation. It was found, however, that actually there was a slight curvature below about 50 volts for the particular chamber. The average ionization current over any interval is proportional to $\Delta V / \Delta t$, the voltage increment over the time interval. Thus, plotting $\Delta V / \Delta t$ against $V$ we may obtain a saturation curve for the chamber in question. Such a curve for a Glasser chamber is shown in Figure 8.

18 The ionization current is proportional to the loss of potential of the system per unit time. The tension of the electrometer fiber is adjusted to give a full-scale deflection for any voltage used on the chamber so so that when exposed to the same radiation, for equal scale deflections the time is less, the lower the fullscale voltage on the system.

${ }_{19} \mathrm{H}$. Fricke and O. Glasser, Am. J. Roent., 13, 453; 1925.

20 R. Braun and H. Kustner, Strahlentherapie, 32, pp. 550 and 739; 1929. 
There is considerable error in obtaining such a curve, but the trend of the points indicates beyond any question that there is not complete saturation below 50 volts.

\section{VI. "WALL EFFECT" IN THIMBLE CHAMBERS}

It has been well recognized that, for most small ionization chambers, the "wall effect" has a very decided influence upon the ionization measured..$^{21}{ }^{22}$ It was this effect which prompted Fricke and Glasser ${ }^{23}$ to develop the "air wall" chamber in which the walls are made up of a compound having the same effective atomic number as air, and in which the ionization is closely parallel to that in an unrestricted volume of air. However, the extensive use of chambers having walls of graphite, magnesium, horn, celluloid, etc., which do not fulfill this condition makes it necessary to establish some correction factor

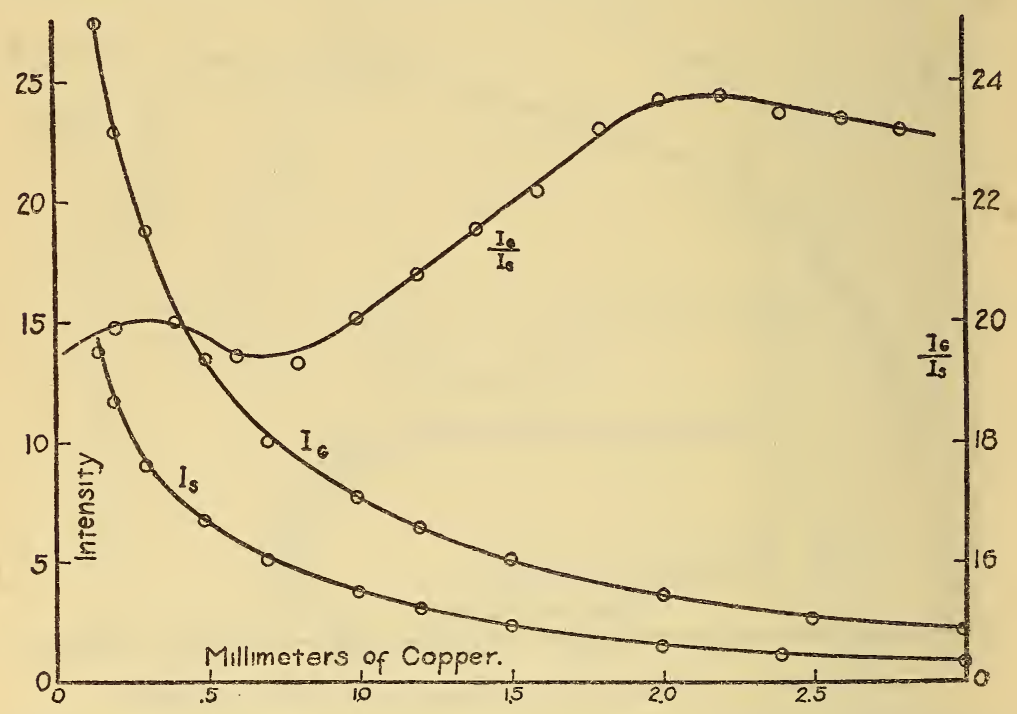

FIGURE 9.-Dependence of measured ionization in a thimble chamber as a function of filtration

for the wall effects. Behnken ${ }^{24}$ applies a correction determined by the half-value layer in copper for each kind of radiation used. His method is probably sufficiently accurate and there seems little chance of improvement unless a standard source of $\mathrm{X}$ rays in which there is a fixed energy distribution is established This might be approached by using constant potential on a tube of somewhat special but nevertheless simple construction. However, it does not seem warranted at this time to introduce the further complication of a standard source of $\mathrm{X}$ rays.

To show the deviation introduced by the wall effect comparisons were made against the standard chamber as indicated in Figures 9 and 10. The Glasser chamber is made of graphite while the Fried-

21 Otto Glasser and U. V. Portmann. (See footnote 9.)

$22 \mathrm{H}$. Behnken. (See footnotes 10 and 12.)

${ }^{23} \mathrm{H}$. Fricke and O. Glasser, Am. J. Roent., 13, p. 462; 1925.

24 H. Behnken, Strahlentherapie, 29, p. 192; 1928. 
rich chamber is horn coated with graphite, these being representative of all of the small chambers available. They were each used with the same electroscope- a modification of the Lutz-Edelman, constructed at the Bureau of Standards. Readings were taken alternately with those of the standard chamber, the X-ray tube being shifted from one system to the other between readings.

Observations may be made in two ways, depending upon the kind of X-ray equipment and the control available. The first method is to maintain a given filter in the beam and vary the voltage, and the second is to maintain the voltage constant and change the filter. In both cases the ionization readings of the two chambers are compared.

Figure 9 shows 2 copper absorption curves made with the standard and the Glasser chambers, respectively, at a tube potential of about $160 \mathrm{kv}$. On the same chart is shown the ratio $I_{g} / I_{s}$ obtained from

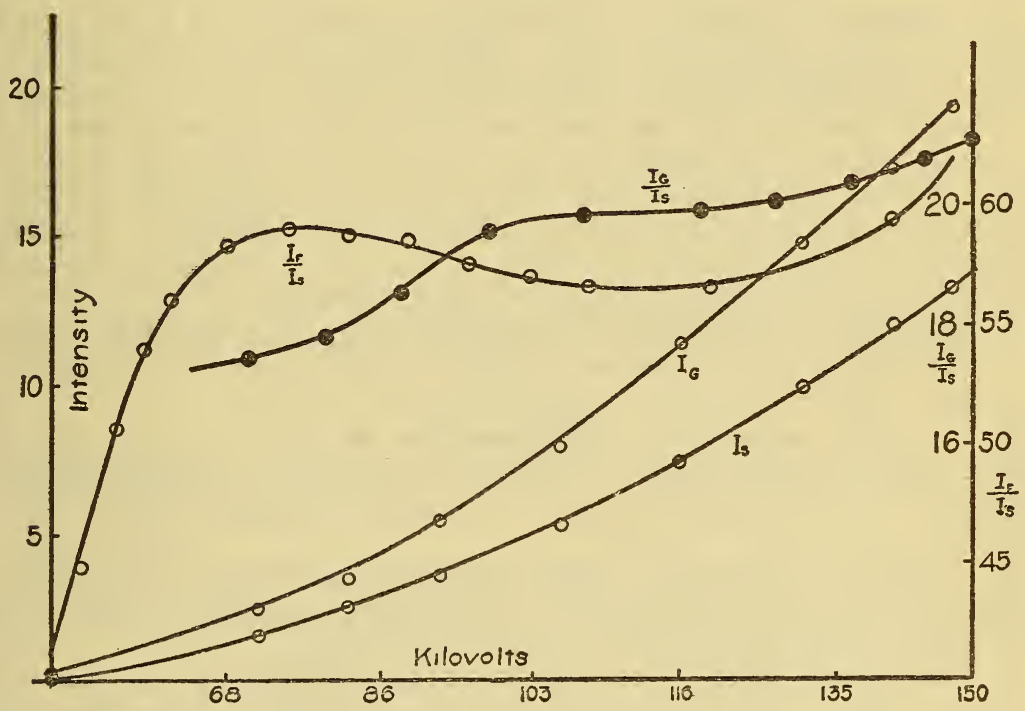

FIGURE 10.-Dependence of measured ionization in a thimble chamber as a function of voltage applied to $X$-ray tube

the curves $I_{g}$ and $I_{s}$. It is seen that this ratio varies from about 19.5 to 23.7 , or about 9.5 per cent from the mean. If we could know that at any point the ratio was correct then a simple correction for the remaining points could be made. Practically, however, this is impossible. Again, for the same chamber the filter was fixed at $0.6 \mathrm{~mm}$ copper and the voltage varied over a wide range. Figure 10 gives the corresponding $I_{g}$ and $I_{s}$ curves and again the ratio $I_{g} / I_{s}$. In this case the ratio varies from 17 to 21 , or about 10 per cent from a mean value. As would be expected in both cases the greatest variation occurs at low voltages and small filtration, under which conditions there is a large amount of soft radiation in the beam. It should be pointed out that in the $I_{g} / I_{s}$ curves there may be an error of 2 or 3 per cent, since the ratio is obtained from two sets of experimental data each having a small error, and since there may also be some error in plotting and reading the 
curves. The importance of the curves lies in showing the complete lack of parallelism in the ionization as measured by the two methods.

In order to obtain some idea of the relation between the measured ionization in two different thimble chambers Figure 10 also shows a curve of $I_{F S} / I$ for a Friedrich chamber under identical conditions as for the Glasser chamber. It will only be pointed out that the form of the $I_{F} / I_{s}$ curve is quite different, as might be expected from the differences in construction between the two chambers.

The most important result of the investigation has been to show the inadequacy of the thimble ionization chamber as a medium through which to compare the fundamental standards in various laboratories. An alternative method has been under investigation for several months and will be described in detail at an early date.

\section{SUMIMARY}

As indicated in the body of the paper there are five factors which must be taken into consideration when calibrating a "Fingerhut" or thimble ionization chamber against an accepted form of standard air ionization chamber. A summary of the possible errors follows:

1. That due to variable energy distribution across the standard $\mathrm{X}$-ray beam. In general, a standard beam will have but a narrow region in its center where the intensity is nearly uniform. Improper placement of the thimble chamber in this beam tends to make the thimble chamber indicate too low X-ray intensity.

2. When the uniform area of the X-ray beam is too small to entirely cover the thimble chamber too low X-ray intensity will, in general, be indicated by the ionization measured in the chamber.

3 . Less than saturation voltage on the thimble chamber will also indicate too low intensity. (Saturation is usually assured in medical dosage meters, but not always in the case of standardization dosage meters.)

4. Improper application of the inverse square law applied in standardizing the thimble chamber will, in general, produce an error in the direction of measuring too low X-ray intensity.

5. The "wall effect" in any but the "Fricke-Glasser air wall chamber" may produce an error in either direction, depending upon the particular chamber used. In general, this effect is so pronounced that an accurate calibration is obtainable only under identical conditions regarding filtration, tube potential, and wave form of the generating equipment.

Some of these factors are of little importance when calibrating a dosage meter which is to be used only for medical purposes. However, if such a dosage meter is to be used for standardizing purposes or for comparing different standards, each factor must be carefully considered.

Washington, June 30, 1929. 